\title{
L-Serine Decreases Taurine Concentration in the Extracellular Fluid of Brain Slices
}

\author{
Kazutaka Shigemi $^{1}$, Koji Tanaka ${ }^{1}$, Kohsuke Hayamizu ${ }^{2,4}$, Donald Michael Denbow ${ }^{3}$, Mitsuhiro Furuse ${ }^{1}$ \\ ${ }^{1}$ Laboratory of Regulation in Metabolism and Behavior, Graduate School of Bioresources and Bioenvironmental Sciences, Kyushu \\ University, Fukuoka, Japan; ${ }^{2}$ FANCL Research Institute, Yokohama, Japan; ${ }^{3}$ Department of Animal and Poultry Sciences, Virginia \\ Tech, Blacksburg, USA; ${ }^{4}$ Human Life Science R\&D Center, Nippon Suisan Kaisha, Tokyo, Japan. \\ E-mail: furuse@brs.kyushu-u.ac.jp
}

Received March $30^{\text {th }}, 2011$; revised May 24 ${ }^{\text {th }}$, 2011; accepted July $10^{\text {th }}, 2011$.

\begin{abstract}
L-Serine is considered a functional amino acid in the central nervous system, and induces sedation and hypnotic effects in some animal models of acute and chronic stress. Accordingly, while L-serine is a candidate anti-stress factor, the central mechanism of L-serine is not clear. The present study clarifies the action of L-serine using acute chick brain slices. We investigated the changes in some extracellular fluid amino acid concentrations in response to $L$-serine perfusion. Taurine concentration decreased while L-alanine concentration increased following L-serine perfusion. To examine the involvement of the taurine transporter, the effect of $L$-serine on the taurine concentration in the presence and absence of $\mathrm{Na}^{+}$was also investigated. $\mathrm{Na}^{+}$had no effect on taurine concentration induced by L-serine perfusion. These results suggest that $L$-serine has an ability to promote L-alanine synthesis facilitating the catabolism of taurine. In conclusion, L-serine modifies the metabolism of taurine and L-alanine in the extracellular space in chick brain.
\end{abstract}

Keywords: L-Serine, Chick, Brain Slice, Taurine, L-Alanine

\section{Introduction}

L-Serine, a non-essential amino acid, is an important metabolic precursor in the synthesis of proteins, sphingolipids, other amino acids and nucleotides [1]. Serine-deficiency syndrome, a congenital disease associated with an inborn error endogenous L-serine biosynthesis suggests that L-serine is vital for humans. Patients with this syndrome suffer from serious neurological symptoms such as congenital microcephaly, seizures and severe psychomotor retardation [2]. However, these symptoms are treatable with oral supplementation of L-serine alone or in combination with glycine [2-4]. Furthermore, results from phosphoglycerate dehydrogenase (Phgdh) null knockout mice show that the Phgdh-dependent pathway of de novo synthesis is the principal source of L-serine in developing embryos. This pathway is essential for normal embryonic development, especially for brain morphogenesis [5]. Mitoma et al. [6] reported that L-serine increased the viability and promoted the growth of dendrites in cultured rat hippocampal neurons. These facts indicate the importance of L-serine in the central nervous system (CNS).

It has been reported that externally supplemented L- serine has anti-stress effects in animals. Asechi et al. conducted a series of experiments to investigate the relationship between L-serine in the CNS and stress-related behavior of animals using the chick separation-stress paradigm [7]. Intracerebroventricular (i.c.v.) injection of Lserine and its derivatives glycine and L-cysteine induced sedative and hypnotic effects under isolation-induced stress in neonatal chicks. Further, it was reported that daily intake of L-serine attenuated symptoms induced by isolation stress in rats [8]. However, the precise mechanism by which administered L-serine induces anti-stress like effects was unclear.

In the mammalian CNS, most of the L-serine is synthesized via a route known as the phosphorylated L-serine pathway [9-12]. In this pathway, L-serine is converted from L-phosphoserine by phosphoserine phosphatase [13]. The L-serine precursor L-phosphoserine has been proposed as a possible endogenous agonist or coagonist at the Group Ш metabotropic glutamate receptors [14], while the L-serine metabolite $\mathrm{D}$-serine is a co-agonist at the $N$-methyl- $D$-aspartate (NMDA) subtype of the ionotropic glutamate receptor [15]. On the other hand, L-serine is supposed to act as an agonist of the $\mathrm{GABA}_{\mathrm{A}}$ receptor in the chick brain [16], and L-serine converted to gly- 
cine acts as an inhibitory neurotransmitter. These facts suggest that L-serine and other substances related to Lserine metabolism have neurochemical effects, and these effects can be either excitatory or inhibitory. In either case, an increase of L-serine by L-serine supplementation can influence brain function as a result of modulation of substances through these metabolic pathways.

Brain slice preparations are well established models for a wide spectrum of in vitro investigations in neuroscience. The action of cells can be observed in real time using brain slice cultures. Therefore, to further investigate the role of L-serine in the chick brain, we examined the effect of L-serine on the external environment of cells using acute brain slices.

\section{Methods}

\subsection{Animals}

One-day-old male layer chicks (Julia) purchased from a local hatchery (Murata Hatchery, Fukuoka, Japan) were maintained in a windowless room at a constant temperature of $30^{\circ} \mathrm{C} \pm 1^{\circ} \mathrm{C}$. Lighting was provided continuously. Chicks were given free access to a commercial starter diet (Toyohashi Feed and Mills Co. Ltd., Aichi, Japan) and water. This experiment was performed at 7 or 8 daysof-age. Experimental procedures followed the guidance for Animal Experiments in the Faculty of Agriculture and in the Graduate Course of Kyushu University and the Law (No. 105) and Notification (No. 6) of the Japanese Government. During the experiments, all efforts were made to minimize animal suffering.

\subsection{Preparation of Drugs}

The standard artificial cerebrospinal fluid (ACSF) was composed of $125 \mathrm{mM} \mathrm{NaCl}, 3 \mathrm{mM} \mathrm{KCl}, 1.25 \mathrm{mM}$ $\mathrm{NaH}_{2} \mathrm{PO}_{4}, 1.5 \mathrm{mM} \mathrm{MgSO}, 1.6 \mathrm{mM} \mathrm{CaCl}, 26 \mathrm{mM} \mathrm{Na}-$ $\mathrm{HCO}_{3}, 10 \mathrm{mM}$ glucose, $500 \mu \mathrm{M}$ ascorbic acid, and 1-5 $\mu \mathrm{M}$ taurine ( $1 \mu \mathrm{M}$ for Experiment $1 ; 5 \mu \mathrm{M}$ for Experiments 2 and 3), prepared with ultrapure water and bubbled with $95 \% \mathrm{O}_{2} / 5 \% \mathrm{CO}_{2}$. Based on previous studies investigating the effects of L-serine in chicks exposed to acute stressful condition [7,16], ACSF containing $84 \mathrm{mM}$ L-serine was used as the L-serine solution in Experiments 1 and 2 .

In Experiment 3, ACSF containing $8.4 \mathrm{mM}$ L-serine was used to confirm whether the effect of L-serine occurred at lower doses. The osmotic pressure was equalized between standard ACSF and the L-serine solution. A $\mathrm{Na}^{+}$-free solution was made by substituting $\mathrm{NaCl}$ and $\mathrm{NaHCO}_{3}$ with choline chloride and $\mathrm{NaH}_{2} \mathrm{PO}_{4}$ with $\mathrm{KH}_{2} \mathrm{PO}_{4}$ in ACSF.

The derivatizing solution (othophthaldialdehyde/2-mercaptoethanol (OPA/2-ME) solution) contained $27 \mathrm{mg}$ of OPA (Wako, Tokyo, Japan) dissolved in $1.0 \mathrm{ml}$ of abso- lute ethanol. After the addition of $20 \mu \mathrm{l}$ of 2-ME (Wako, Tokyo, Japan) the solution was diluted to $10 \mathrm{ml}$ with 0.1 mol/l carbonate buffer ( $\mathrm{pH}$ 9.5). Further, this solution was diluted with $0.1 \mathrm{~mol} / 1$ carbonate buffer $(1: 4)$ before use.

\subsection{Brain Slice Preparation}

Chicks were decapitated after cervical dislocation. The brains were quickly removed and placed in ice-cold ACSF. Vertical telencephalic slices $500 \mu \mathrm{m}$ in thickness $(2,500$ $3,000 \mu \mathrm{m}$ from anterior end of telencephalon) were immediately prepared using a Leica VT1000S microtome ${ }^{\circledR}$ (Leica, Nussloch, Germany), and kept in an interfacetype holding chamber at $38^{\circ} \mathrm{C}$ for at least $30 \mathrm{~min}$ in ACSF before applying each treatment solution.

\subsection{Perfusion Manipulation}

After a $30 \mathrm{~min}$ recovery period, brain slices were exposed to each solution during a recording period and the perfusate was collected for $20 \mathrm{~min}$. The L-serine solution was perfused for $5-10 \mathrm{~min}$ in this period. The flow rate was set at $1.5 \mathrm{ml} / \mathrm{min}$. In Experiments 1 and 3, amino acid concentrations of collected perfusate were analyzed. In Experiment 2, the free amino acid content of telencephalic slices was measured in slices collected and preserved in liquid nitrogen after the recording period.

\subsection{Amino Acid Analysis}

The concentrations of 8 free amino acids including Laspartate, L-glutamate, L-glutamine, glycine, L-serine, taurine, L-alanine, and GABA were determined by the method described below. The tissue samples were homogenized in ice-cold $0.2 \mathrm{M}$ perchloric acid solution containing $0.01 \mathrm{mM}$ EDTA $2 \mathrm{Na}$ and left for deproteinization on ice. After $30 \mathrm{~min}$, the mixtures were centrifuged at $18,000 \mathrm{xg}$ for $15 \mathrm{~min}$ at $0^{\circ} \mathrm{C}$ and the resultant supernatants then adjusted to $\mathrm{pH} 7$ with $1 \mathrm{M}$ sodium hydroxide. These solutions were diluted by $0.5 \%$ with $10 \%$ methanol. The diluted samples were filtered through a $0.22 \mu \mathrm{m}$ filter (Millipore, Bedford, USA) and were applied for high-performance liquid chromatography (HPLC) after the derivation as described below. The pellets were used for protein assay using a commercially available kit (BCA Protein Assay $\mathrm{Kit}^{\circledR}$, Thermo Fisher Scientific Inc, Rockford, USA).

Collected perfusate samples were centrifuged with a centrifuge-filtration unit (Millipore) at 10,000 $\mathrm{g}$ for $5 \mathrm{~min}$ at $0^{\circ} \mathrm{C}$. A $60 \mu \mathrm{l}$ aliquot of filtrate was mixed with $20 \mu \mathrm{l}$ of the OPA/2-ME solution and then the samples were incubated at room temperature for $2.5 \mathrm{~min}$. The mixture (30 $\mu \mathrm{l})$ was applied to an HPLC system (Eicom, Kyoto, Japan) with a $150 \times 2.1 \mathrm{~mm}$ octadecyl silane column $\left(\mathrm{SC}-5 \mathrm{ODS}^{\circledR}\right.$, Eicom) with an electrochemical detector (ECD-300 ${ }^{\circledR}$, 
Eicom) at an applied potential of $+600 \mathrm{mV}$ versus $\mathrm{Ag} / \mathrm{AgCl}$ reference analytical electrode. Changes in electric current (nA) were recorded in a computer using an interface system Power Chrom $^{\circledR}$ (ver 2.3.2.j, AD Instruments, Tokyo, Japan). The mobile phase consisted of 0.1 $\mathrm{mol} / \mathrm{l}$ monosodium phosphate buffer ( $\mathrm{pH} 6.0$ ), methanol (7:3) containing $5 \mathrm{mg} / 1$ EDTA2Na at a flow rate of 0.23 $\mathrm{ml} / \mathrm{min}$.

\subsection{Statistical Analysis}

The perfusate data were analyzed using a paired t-test in Experiment 1, or two-way repeated measure ANOVA in Experiment 3. A Tukey-Kramer test was done as a post hoc test. The data for slice samples were analyzed using a t-test. Values are presented as means \pm S.E.M. Statistical analysis was conducted using a commercially available package StatView ${ }^{\circledR}$ (version 5, SAS Institute, Cary, USA, 1998).

\section{Results}

The effects of L-serine on the concentration of taurine and L-alanine in the perfusate are shown in Figure 1.

The addition of L-serine significantly decreased the concentration of taurine $(P<0.0001)$ and increased the concentration of L-alanine $(P<0.0001$ in the perfusate. Other amino acids including aspartate, glutamate, and glycine did not change (data not shown). GABA concentrations were below the level of detection.

The effects of L-serine on the concentration of free taurine in the telencephalic slices exposed to $84 \mathrm{mM} \mathrm{L}$ serine were determined. The taurine concentration (control, $86.559 \pm 7.637 \mathrm{nmol} / \mathrm{mg}$ protein; L-serine, 100.367 $\pm 6.161 \mathrm{nmol} / \mathrm{mg}$ protein, respectively) did not change significantly in the slices after exposure to L-serine $(P>$ 0.05).

Using a lower concentration of L-serine $(8.4 \mathrm{mM}$, also decreased $[F(2,36)=36.819, P<0.0005]$ the concentration of taurine in the perfusate (Figure 2). Even though $\mathrm{Na}^{+}$was deleted from the perfusate, the effect of L-serine was still evident.

\section{Discussion}

According to Hájos et al. [17], taurine is needed in the perfusate to facilitate volume regulation and maintain ATP concentration of brain slices in culture. In this study, we clarified that taurine in the perfusate was dramatically reduced when chick telencephalic slices were exposed to $84 \mathrm{mM} \mathrm{L}$-serine (Figure 1). This result implies that Lserine promotes taurine uptake from the perfusate via a taurine transporter or taurine is metabolized by the addition of L-serine. To confirm the former hypothesis, we first determined the free taurine concentration in telencephalic slices after exposure to L-serine. The concentra-
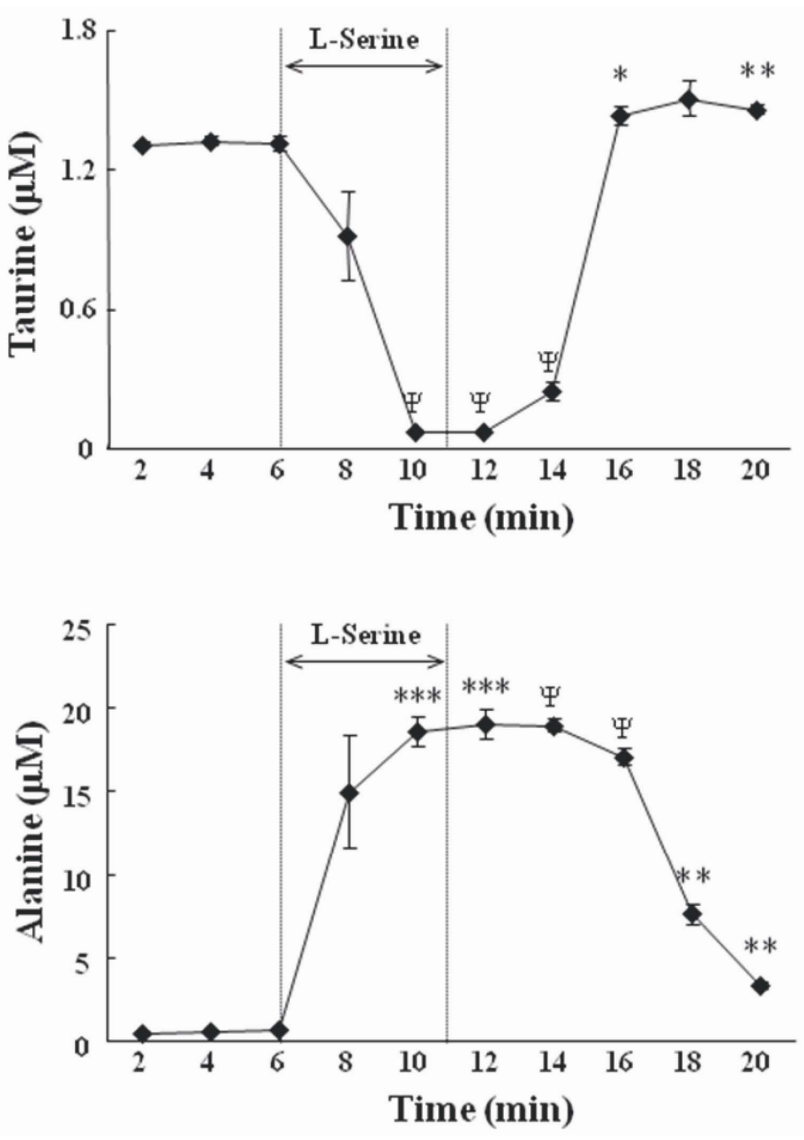

Figure 1. Changes of the concentration of taurine (upper panel) and alanine (lower panel) in the perfusate when brain slices were exposed to $84 \mathrm{mM}$ L-serine containing artificial cerebrospinal fluid (ACSF). Results are expressed as means \pm S.E.M. A significant difference from the value at 2 min is denoted by the asterisk $(*, P<0.05 ; * *, P<0.005$; $* * *, P<0.0005 ; \psi, P<0.0001$; paired t-test). The number of brain samples used for analysis was 4 . Taurine $(1 \mu \mathrm{M})$ was added to the perfusate in advance to maintain viability of slices. L-Serine was added for 5 min.

tion of taurine in telencephalic slices was not increased by exposure to L-serine.

Secondly, we examined whether taurine was decreased in the perfusate when $\mathrm{Na}^{+}$was deleted because it is known that the uptake of taurine via the taurine transporter is a $\mathrm{Na}^{+}$-dependent system [18]. The background level of taurine was higher in $\mathrm{Na}^{+}$free conditions than in the normal $\mathrm{Na}^{+}$environment (Figure 2). This suggests that the taurine transporter activity was inhibited by $\mathrm{Na}^{+}$depletion and that the taurine level in the extracellular fluid equilibrated at higher levels than in the normal $\mathrm{Na}^{+}$condition. In contrast, alteration of taurine in the perfusate by the addition of L-serine was not influenced in the $\mathrm{Na}^{+}-$ free environment. These data indicate that the decrease of taurine in the perfusate was not induced by the uptake of 


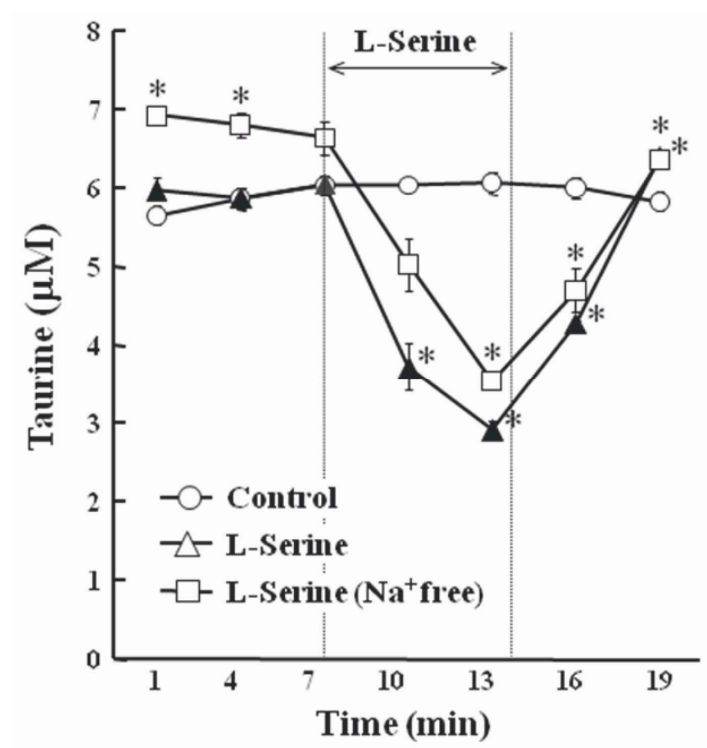

Figure 2. Changes of the concentration of taurine in the perfusate when brain slices were exposed to $8.4 \mathrm{mM}$ L-serine containing artificial cerebrospinal fluid (ACSF). Results are expressed as means \pm S.E.M. The number of samples used for analysis was 3 . Taurine $(5 \mu M)$ was added to the perfusate. L-Serine was added for $7 \mathrm{~min}$. The significant difference from control group is denoted by the asterisk $(P$ $<0.05)$.

taurine via the $\mathrm{Na}^{+}$dependent taurine transporter from the perfusate into slices.

It is known that taurine plays an important role in the regulation of cell volume of neurons and glia when the osmolality between intracellular fluid and extracellular fluid is different. Taurine is transported via the cell membrane by the taurine transporter depending on the change in osmotic pressure [18]. It is possible that the change in taurine in the perfusate was due to the osmotic change by switching the perfusate from ACSF to the L-serine-containing fluid. However, in the present study, this was not the case because taurine was also decreased by L-serine when the osmoality of the perfusate remained constant. So, the change in taurine in the perfusate was not due to osmotic changes by the addition of L-serine. This reinforces the notion that the taurine reduction in the perfusate was not induced by the uptake of taurine from the perfusate into slices, and we should consider the possibility that taurine in the perfusate was metabolized by the addition of L-serine, although there remains the slight possibility that taurine was transported via a $\mathrm{Na}^{+}$independent system.

In the present study, we also observed increased Lalanine concentrations in the perfusate when the brain slice was exposed to $84 \mathrm{mM}$ L-serine (Figure 1). This implies that L-alanine synthesis was promoted by L-serine since L-serine is indirectly metabolized to L-alanine via pyruvate (Figure 3(a)). In the mammalian CNS, most L-serine is synthesized via a route known as the phosphorylated L-serine pathway [9-12], and it is known that the regulation of the enzymes responsible for serine production and metabolism has been linked to adaptations to dietary protein excess and protein restriction [19]. It has been reported that the expression of 3-phosphoglycerate dehydrogenase (3-PGDH), the enzyme representing the "committed step" in L-serine bio synthesis, is up-regulated in response to a low protein diet [20-22]. In addition, the activities of the other enzymes in this pathway, phosphoserine aminotransferase (PSAT) and phosphoserine phosphatase (PSP), have also been shown to be up-regulated in response to a low protein diet $[23,24]$. Further, a down regulation of serine dehydrogenase, one of the enzymes catalyzing L-serine synthesis, induced by amino acid-deficient diets has also been reported [20,22,25].

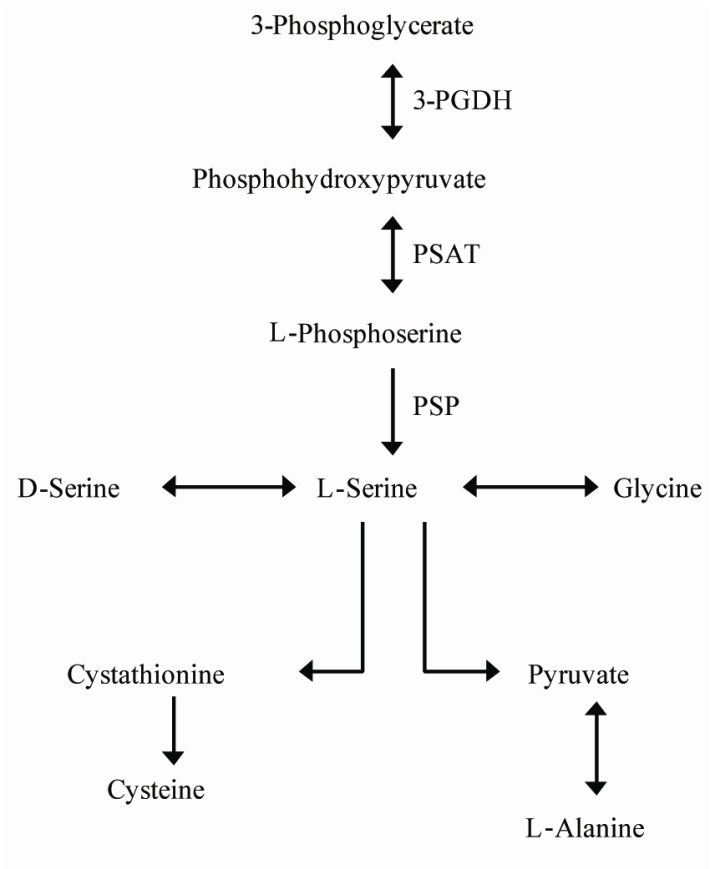

(a)

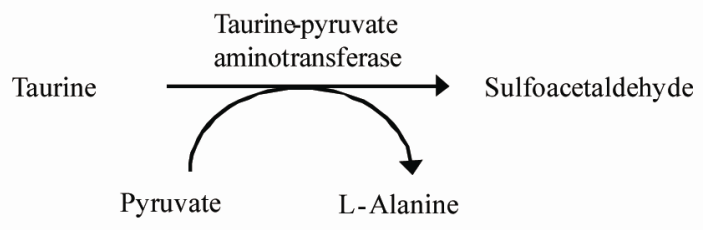

(b)

Figure 3. (a) Pathways for the biosynthesis and catabolism of L-serine (refer to Antiflick et al., [9]). (b) Degradative pathway of taurine by taurine-pyruvate aminotransferase [EC: 2.6.1.77]. 
These facts suggest that L-serine metabolism is tightly regulated in response to the abundance of L-serine to prevent a lack of L-serine, and it is reasonable to hypothesize that the catabolism process of L-serine is promoted when L-serine is abundant in chick brain. L-alanine synthesis, which occurs downstream of L-serine metabolism (Figure 3(a)), may also be upregulated by L-serine, and L-alanine in the perfusate was increased in the present study.

It was reported that L-serine and L-alanine were rapidly released into the culture medium whereas other amino acids (glycine, L-aspartic acid, L-asparagine, Lproline) were not changed when the culture of hippocampal astrocytes of mammal was maintained for 7 days [6]. This suggests that L-serine and L-alanine synthesis in the brain can be linked, although neurotrophic activity was investigated only following L-serine [6]. In the present study, glycine in the perfusate did not change following L-serine suggesting that L-serine has a tendency to be metabolized to L-alanine but not glycine. Recently, Kurauchi et al. [26] reported that central L-alanine caused a hypnotic effect in chicks exposed to an acute stressful condition. Therefore, increased L-alanine in the brain can bring about a positive effect to resist stress.

In considering the mechanism by which L-serine promotes taurine catabolism, the pathway of taurine metabolism in the brain is important. For many years, taurine was known to be an end product of the metabolism of sulphur-containing amino acids, although physiological effects were not well understood. However, taurine catabolism was indicated by the finding of isethionic acid in the rat brain [27]. The most likely intermediate in the conversion of taurine to isethionate would be sulphoacetaldehyde. Although the presence of this aldehyde has never been reported in any mammalian oravian system, it has been shown to be produced from taurine by enzymecatalyzed processes in a number of bacteria. The conversion is catalyzed by transaminases in Achromobacter superficialis [28] and Pseudomonas aeruginosa [29] and by a taurine dehydrogenase in Rhodopseudomonas palustris from taurine [30]. The enzyme taurine-pyruvate aminotransferase [EC: 2.6.1.77] catalyzes the transamination of taurine with pyruvate as the amino-group acceptor to sulfoacetaldehyde and L-alanine (Figure 3(b)). Although taurine-pyruvate aminotransferase has never been identified in any vertebrate, the finding that the formation of sulfoacetaldehyde from taurine can occur in mammalian systems implies the presence of this enzyme [31]. It appears possible that the L-alanine increases and taurine decreases in the perfusate were linked since these changes might be caused by this metabolite step. In this study, added L-serine promoted the increase of L-alanine via pyruvate and this step partially involves taurine ca- tabolism by taurine-pyruvate aminotransferase causing a taurine decrease by L-serine in the perfusate. Here, we show the novel probability of the presence of taurinepyruvate aminotransferase in the CNS of chicks.

It was known that taurine injection induces an antianxiety-like effect in mice, and the GABAergic system may be involved in this effect. However, El Idrissi et al. reported that chronic supplementation of taurine was anxiogenic in the elevated plus maze whereas acute injection of taurine suppressed anxiety in mice [32]. This fact suggests that chronic increases of taurine in the brain may induce negative effects on stressful behavior of animals. Therefore, the effect of L-serine to reduce taurine of extracellular fluid may be reasonable to maintain mental status of animals.

L-Serine markedly changes some amino acid concentrations in the extracellular space. These changes may be involved in the mechanisms of the anti-stress effects of L-serine observed in some animal models [7-8].

\section{Acknowledgements}

This work was supported by a Grant-in-Aid for Scientific Research from Japan Society for the Promotion of Science.

\section{REFERENCES}

[1] S. Furuya, "An Essential Role for de Novo Biosynthesis of L-Serine in CNS Development," Asia Pacific Journal of Clinical Nutrition, Vol. 17, Suppl. 1, 2008, pp. 312315 .

[2] J. Jaeken, M. Detheux, L. Van Maldergem, M. Foulon, H. Carchon and E.Van Schaftingen, "3-Phosphoglycerate Dehydrogenase Deficiency: An Inborn Error of Serine Biosynthesis," Archives of Disease in Childhood, Vol. 74, No. 6, January 1996, pp. 542-545. doi:10.1136/adc.74.6.542

[3] T. J. de Koning, M. Duran, L. Van Maldergem, M. Pineda, L. Dorland, R. Gooskens, J. Jaeken and B. T. Poll-The, "Con- genital Microcephaly and Seizures Due to 3-Phospho- Glycerate Dehydrogenase Deficiency: Outcome of Treat- ment with Amino Acids," Journal of Inherited Metabolic Disease, Vol. 25, No. 2, May 2002, pp. 119-125.doi:10.1023/A:1015624726822

[4] T. J. de Koning, L. W. Klomp, A. C. van Oppen, F. A. Beemer, L. Dorland, I. E. T. van den Berg and R. Berger, "Prenatal and Early Postnatal Treatment in 3-PhosphoglyCerate-Dehydrogenase Deficiency," Lancet, Vol. 364. No. 9452, December 2004, pp. 2221-2222.

[5] K. Yoshida, S. Furuya, S. Osuka, J. Mitoma, Y. Shinoda, M. Watanabe, N. Azuma, H. Tanaka, T. Hashikawa, S. Itohara and Y. Hirabayashi, "Targeted Disruption of the Mouse 3-Phosphoglycerate Dehydrogenase Gene Causes Severe Neurodevelopmental Defects and Results in Embryonic Lethality," Journal of Biological Chemistry, Vol. 275, No. 5, January 2004, pp. 3573-3577. 
[6] J. Mitoma, S. Furuya and Y. Hirabayashi, "A Novel Metabolic Communication between Neurons and Astrocytes: Non-Essential Amino Acid L-Serine Released from Astrocytes is Essential for Developing Hippocampal Neurons," Neuroscience Research, Vol. 30, No. 2, February 1998, pp. 195-199. doi:10.1016/S0168-0102(97)00113-2

[7] M. Asechi M, S. Tomonaga, T. Tachibana, L. Han, K. Hayamizu, D. M. Denbow and M. Furuse, "Intracerebroventricular Injection of L-Serine Analogs and Derivatives Induces Sedative and Hypnotic Effects under an Acute Stressful Condition in Neonatal Chicks," Behavioural Brain Research, Vol. 170, No. 1, June 2006, pp. 71-77. doi:10.1016/i.bbr.2006.02.005

[8] K. Shigemi, Y. Tsuneyoshi, S. Yamada, Y. Kabuki, K. Hayamizu, D. M. Denbow and M. Furuse, "Oral Administration of L-Serine Reduces the Locomotor Activity of Socially Isolated Rats," Neuroscience Letters, Vol. 468, No. 1, January 2010, pp. 75-79. doi:10.1016/j.neulet.2009.10.068

[9] J. E. Antflick, G. B. Baker and D. R. Hampson, "The Effects of a Low Protein Diet on Amino Acids and Enzymes in the Serine Synthesis Pathway in Mice," Amino Acids, Vol. 39, No. 1, June 2010, pp. 145-153. doi:10.1007/s00726-009-0387-8

[10] M. Lowry, D. E. Hall, M. S. Hall and J. T. Brosnan, "Renal Metabolism of Amino Acids in Vivo: Studies On Serine and Glycine Fluxes," American Journal of Physiology, Vol. 252, No. 2, February 1987, pp. F304-F309.

[11] D. A. Fell and K. Snell, "Control Analysis Of Mammalian Serine Biosynthesis. Feedback Inhibition on the Final Step," Biochemical Journal, Vol. 256, No. 1, November 1988, pp. 97-101.

[12] K. Snell and D. A. Fell, "Metabolic Control Analysis of Mam-Malian Serine Metabolism," Advances in Enzyme Regulation, Vol. 30, 1990, pp. 13-32. doi:10.1016/0065-2571(90)90006-N

[13] J. F. Collet, V. Stroobant and E. V. Schaftingen, "Mechanistic Studies of Phosphoserine Phosphatase, an Enzyme Related to p-Type ATPases," Journal of Biological Chemistry, Vol. 274, No. 48, November 1999, pp. 3398533990. doi:10.1074/jbc.274.48.33985

[14] J. E. Antflick, S. Vetiska, J. S. Baizer, Y. Yao, G. B. Baker and D. R. Hampson, "L-Serine-O-Phosphate in the Central Nervous System," Brain Research, Vol. 1300, December 2009, pp. 1-13. doi:10.1016/j.brainres.2009.08.087

[15] A. Panatier, D. T. Theososis, J. P. Mothet, B. Touquet, L. Pollegioni, D. A. Poulain and S. H. R. Oliet, "Glia-Derived D-Serine Controls NMDA Receptor Activity and Synaptic Memory," Cell, Vol. 125, No. 4, May 2006, pp. 775-784.

[16] K. Shigemi, Y. Tsuneyoshi, K. Hamasu, L. Han, K. Hayamizu, D. M. Denbow and M. Furuse, "L-Serine Induces Sedative and Hypnotic Effects Acting at GABAA Receptors in Neonatal Chicks," European Journal of Pharmacology, Vol. 599, No. 1-3, December 2008, pp. 86-90. doi:10.1016/j.ejphar.2008.09.036
[17] N. Hájos and I. Mody, "Establishing a Physiological Environment for Visualized in Vitro Brain Slice Recordings by Increasing Oxygen Supply and Modifying aCSF Content," Journal of Neuroscience Methods, Vol. 183, No. 2, October 2009, pp. 107-113. doi:10.1016/i.jneumeth.2009.06.005

[18] I. H. Lambert, "Regulation of the Cellular Content of the Organic Osmolyte Taurine in Mammalian Cells," Neurochemical Research, Vol. 29, No. 1, January 2004, pp. 27 63.

[19] Y. Noguchi, N. Shikata, Y. Furuhata, T. Kimura and M. Takahashi, "Characterization of Dietary Protein-Dependent Amino Acid Metabolism by Linking Free Amino Acids with Transcriptional Profiles through Analysis of Correlation," Physiological Genomics, Vol. 34, No. 3, August 2008, pp. 315-326.

doi:10.1152/physiolgenomics.00007.2008

[20] J. Mauron, F. Mottu and G. Spohr, "Reciprocal induction and repression of Serine Dehydratase and Phosphoglycerate Dehydrogenase Bby Proteins and Dietary-Essential Amino Acids in Rat Liver," European Journal of Biochemistry, Vol. 32, No. 2, January 1973, pp. 331-342. doi:10.1111/j.1432-1033.1973.tb02614.x

[21] Y. Achouri, M. Robbi and E. Van Schaftigen, "Role of Cysteine in the Dietary Control of the Expression of 3-Phosphoglycerate Dehydrogenase in Rat Liver," Biochemical Journal, Vol. 344, November 1999, pp. 15-21.

[22] K. Nagao, M. Bannai, S. Seki, M. Mori and M. Takahashi, "Adaptational Modification of Serine and Threonine Metabolism in the Liver to Essential Amino Acid Deficiency in Rats," Amino Acids, Vol. 36, No. 3, March 2009, pp. 555-562. doi:10.1007/s00726-008-0117-7

[23] K. Lund, D. K. Merrill and R. W. Guynn, "The Reactions of the Phosphorylated Pathway of L-Serine Biosynthesis: Thermodynamic Relationships in Rabbit Liver in Vivo," Archives of Biochemistry and Biophysics, Vol. 237, No. 1, February 1985, pp. 186-196. doi:10.1016/0003-9861(85)90268-1

[24] R. W. Guynn, D. K. Merrill and K. Lund, "The Reactions of the Phosphorylated Pathway of L-Serine Biosynthesis: Thermodynamic Relationships in Rat Liver in Vivo," Archives of Biochemistry and Biophysics, Vol. 245, No. 1, February 1986, pp. 204-211.

[25] H. H. Xue, M. Fujie, T. Sakaguchi, T. Oda, H. Ogawa, N. M. Kneer, H. A. Lardy and A. Ichiyama, "Flux of the L-Serine Metabolism in Rat Liver," Journal of Biological Chemistry, Vol. 274, No. 23, June 1999, pp. 1602016027. doi: $10.1074 / \mathrm{jbc} .274 .23 .16020$

[26] I. Kurauchi, H. Yamane, Y. Tsuneyoshi, D. M. Denbow and M. Furuse, "Central L-Alanine Reduces Energy Expenditure with a Hypnotic Effect under an Acute Stressful Condition in Neonatal Chicks," Amino Acids, Vol. 36, No. 1, January 2009, pp. 131-135. doi:10.1007/s00726-008-0042-9

[27] E. J. Peck and J. Awapara, "Formation of Taurine and Isethionic Acid in Rat Brain," Biochimica et Biophysica Acta, Vol. 141, No. 3, August 1967, pp. 499-506. 
[28] K. Yonaha, S. Toyama and K. Soda, "Taurine-Glutamate Tranaminase," Methods in Enzymology, Vol. 113, 1985, pp. 102-108. doi:10.1016/S0076-6879(85)13023-5

[29] G. Shimamoto and R. S. Berk, "Catabolism of Taurine in Pseudomonas Aeruginosa," Biochimica et Biophysica Acta, Vol. 569, No. 2, August 1979, pp. 287-292.

[30] K. Denger, S. Weinitschke, K. Hollemeyer and A. M. Cook, "Sulfoacetate Generated by Rhodopseudomonas Palustris from Taurine," Archives of Microbiology, Vol. 182, No. 2-3, October 2004, pp. 254-258. doi:10.1007/s00203-004-0678-0
[31] C. Cuningham, K. F. Tipton and H. B. F. Dixon, "Conversion of Taurine into N-Chlorotaurine (Taurine Chloramine) and Sulphoacetaldehyde in Response to Oxidative Stress," Biochemical Journal, Vol. 330, March 1998, pp. 939-945.

[32] A. El Idrissi, L. Boukarrou, W. Heany, G. Malliaros, C. Sangdee and L. Neuwirth, "Effects of Taurine on Anxiety-Like and Locomotor Behavior of Mice," Advances in Experimental Medicine and Biology, Vol. 643, 2009, pp. 207-215. doi:10.1007/978-0-387-75681-3 21 